\section{Emergency physicians' and nurses' perception of quality of elderly care in the Emergency Department. Results of a national survey}

\author{
Gianfranco Cervellin, ${ }^{1}$ Ivo Casagranda, \\ Pierdante Piccioni, ${ }^{3}$ Giorgio Ricci, ${ }^{4}$ \\ Mario Benatti, ${ }^{1}$ \\ Massimo Pesenti Compagnoni ${ }^{5}$ \\ 'Emergency Department, Parma \\ University Hospital; ${ }^{2}$ Emergency \\ Department, SS. Antonio e Biagio e \\ Cesare Arrigo Hospital, Alessandria; \\ ${ }^{3}$ Emergency Department, Lodi Hospital; \\ ${ }^{4}$ Emergency Department, Verona \\ University Hospital; ${ }^{5}$ Emergency \\ Department, Aosta Hospital, Italy
}

\section{Abstract}

The present study has been carried out to evaluate the current status of clinical, educational, social, ethical, and resource issues related to the care of the elderly among emergency physicians (EPs) and emergency nurses (ENs) in Italy. A questionnaire was designed by our scientific society, the Academy of Emergency Medicine and Care (AcEMC), and disseminated directly or by e-mail to a large number of EPs and ENs in several Emergency Departments (EDs) throughout Italy, as well as in a Swiss center. Of the questionnaires, 524 were filled out and returned. Responders were in the majority females [ 273 vs $239 ; 12$ not available (NA)], and nurses [259 vs 207 physicians vs 54 auxiliary; $4 \mathrm{NA}$ ]. Responses to five questions appeared to be normally distributed, whereas other questions elicited interesting responses. In this study focusing for the first time on the EPs' and ENs' perception of a broad spectrum of issues related to the elderly emergency care, the Italian community of EPs and ENs seems to be rather comfortable with geriatric emergency medicine, but largely unsatisfied with healthcare out-of-hospital services. The lack of elderly-specific diagnostic and therapeutic post-discharge pathways is broadly appreciated, and the concept of medical futility is not explicitly included in the vast majority of Italian hospital protocols.

\section{Introduction}

The mean age and the age distribution of the population is constantly increasing in many countries, due to both increased life expectancy and decreased fertility rates. ${ }^{1}$ The elderly also represents a growing concern for Emergency Departments (EDs), accounting for 12 to $24 \%$ of all visits. ${ }^{2}$ This population is admitted to the ED more often than younger adults, and often presents higher level of acuity, more severe medical conditions, a large number of comorbidities, which require a large volume of testing since older patients are more prone to be admitted and are at higher risk of death., ${ }^{3,4}$ Since the number of elderly patients presenting to overcrowded EDs seems to be destined to further increase, the comprehensive care of these patients is emerging as a crucial issue in the field of emergency medicine. ${ }^{3,4}$ Hence, some authors have recently hypothesized that the emergency care offered to elderly patients may be characterized by poorer quality compared to that administered to younger adults. ${ }^{5}$ Although several reports exist on elderly patients' perception and needs in ED care ${ }^{6}$ little is known about emergency physicians'(EPs) and emergency nurses' (ENs) perspective of the geriatric emergency medicine that they are currently practicing. One single survey has been published more than two decades ago, showing that the majority of EPs had more difficulty in managing of older compared with younger patients for each of seven indicative clinical presentations (i.e., abdominal pain, altered mental status, chest pain, dizziness/vertigo, fever, headache, major trauma), and most respondents reported that each of these presentations required more time and resources for management of older patients. ${ }^{6}$ Moreover, the majority of them believed that the time spent during residency training regarding geriatric emergency medicine was inadequate. ${ }^{7}$ It is hence widely acknowledged that both EPs and ENs may have a particularly negative perception of aging, based on the patient population that they usually manage, and this is probably due to the fact that the interaction in the ED may produce a misleading picture of elderly people. ${ }^{8}$

As such, aim of this study was to evaluate the perception of the current status of clinical, educational, social, ethical, and resource issues related to the care of the elderly among EPs and ENs in Italy.

\section{Materials and Methods}

A questionnaire based on 14 issues (Figure 1) was designed by our scientific society [Academy of Emergency Medicine and Care (AcEMC)], and subsequently disseminated either directly or via e-mail to a large number of EPs and ENs in several EDs throughout Italy and in one Swiss center (Bern). For the purposes of this questionnaire, elderly has been defined as a patient of $>80$ years old, or, if aged
Correspondence: Gianfranco Cervellin, Emergency Department, Parma University Hospital, via Gramsci 14, 43126 Parma, Italy. Tel. +39.0521.703800 - Fax: +39.0521.703144. E-mail: gcervellin@ao.pr.it

Key words: elderly, emergency department, survey, quality of care.

Aknowledgements: the Authors acknowledge all members of the National Committee of AcEMC (Libero Barozzi, Mario Cavazza, Daniele Coen, Nicola Di Battista, Carlo Fraticelli, Fabrizio Giostra, Giuseppe Lippi, Carlo Alessandro Locatelli, Ilenia Mezzocolli, Carolina Prevaldi, Giovanni Ricevuti, Bruno Tartaglino) for the kind support in collecting the questionnaires, as well as all doctors and nurses participating in the survey. The results of this study have been partly presented during the AcEMC Congress Geriatric Emergency Medicine held in Verona, Italy, May $16^{\text {th }}-17^{\text {th }}, 2014$.

Received for publication: 24 May 2014

Revision received: 4 August 2014.

Accepted for publication: 11 August 2014.

This work is licensed under a Creative Commons Attribution 3.0 License (by-nc 3.0).

(C) Copyright G. Cervellin et al., 2014

Licensee PAGEPress, Italy

Emergency Care Journal 2014; 10:4173

doi:10.4081/ecj.2014.4173

65-80 years, with defined frailty. For each question, five numeric answers were provided, ranging from 1 (totally disagree) to 5 (totally agree). Several questions (i.e., 1, 2, 5, 7, 8, 11, and 12) were focused on the perception of attitude and capability to face geriatric emergency medicine. Some questions (i.e., 3, 4, 6, 13) were focused on the attitude and/or the quality of care and health records in pre-hospital and in-hospital settings (i.e., other than ED). Two questions (i.e., 9 and 10) were focused on what EPs and ENs consider possible and useful to be implemented in order to improve elderly care. Finally, the last question was focused on a specific topic, i.e. futility. Not surprisingly, this question collected the lowest number of answers (i.e., 451). Anonymous survey responses were collected. For each center, an EP was responsible for the distribution and collection of the questionnaires, as well as for returning the surveys to our secretariat. All the questionnaires were filled out and collected between March and May 2014.

The study was performed in agreement with the ethical standards established by the institution in which the experiments were performed and the Helsinki Declaration of 1975. Due to the intrinsic nature of the study, an approval by ethic committee was not required. Each EP or nurse freely decided to respond or not to the questionnaire. 


\section{Results}

A total of 524 questionnaires were filled out and returned, with a variable number of responses to the single items (i.e., from 9 to 14 , leading to a total of responses varying from 451 to 524). Responders were in majority females [ 273 vs 239; 12 not available (NA)], and nurses (259 vs 207 physicians vs 54 auxiliary; $4 \mathrm{NA}$ ). The age distribution was: $<30 \mathrm{yrs,}$ 44 responders; $30-39$ yrs, 177 responders; 40 49 yrs, 186 responders; $50-59$ yrs, 66 responders; $>60$ yrs, 15 responders; NA, 36 responders. The responses did not show differences related to gender, age or role.

The questions were the following: $1=$ In your $E D$ the elderly care has the same quality of adult care; $2=$ Competence and communication attitudes of emergency health care professionals are the same with elderly and adult patients; $3=$ Health and social recordings of elderly patients referred to the ED by their general practitioners are generally of good quality; $4=$ Health and social recordings of elderly patients referred to the ED by nursing homes are generally of good quality; $5=$ Medical causes for non specific, typically geriatric, presentations (i.e., delirium, falls, hypokynesis) are systematically investigated; $6=$ The willingness to admit elderly patients to medical wards is the same as for adult patients; 7=During ED process basic needs of elderly patients (i.e., meals, hygiene, micturition, evacuation) are always met; $8=$ In your ED objectives tools (i.e., scores) for functional, cognitive and social evaluation of elderly patients are generally used; $9=$ In your opinion in daily routine it would be possible to use objectives tools (i.e., scores) in functional, cognitive and social evaluation of elderly patients; 10=It would be useful to use objectives tools (i.e., scores) for functional, cognitive and social evaluation of elderly patients in daily routine; 11=Before discharging an elderly patient, in your ED a quick functional, cognitive and social evaluation is generally performed; $12=$ The patient age influences by itself the triage priority code, or some different priorities are given to the elderly if the triage code is the same; 13=In your Hospital elderly-specific diagnostic and therapeutic postdischarge pathways are generally provided; $14=$ In your Hospital the concept of futility is explicitly included in protocols.

Answers to question n. 6, 7, 9, 11, 12 seem to be normally distributed (i.e., in a Gauss-like manner), so they are neither displayed nor commented. The distributions of answers to questions $1,2,3,4,5,8,10,13,14$ are significantly skewed towards values 1 or 5 (Figures 2 and 3). Regarding question 8 , due to the intrinsic nature of the study, it was not possible to know which kind of objective tool for functional, cognitive and social evaluation of elderly patients was used by the few responders answering 4 or 5 (i.e., agree or totally agree). There were no significant differences in responses between large and small hospital, or universities centers.

\section{Discussion}

This study was focused for the first time on the EPs' and ENs' perception of a broad spectrum of issues related to elderly emergency care. Interestingly, the vast majority of EPs and ENs perceived that the quality of emergency elderly care is at least good as adult emergency care (71.8\%), and that their competence and communication attitude toward elderly patients was as good as toward adult patients (61.7\%). More than half of responders (52.1\%) declared to always investigate a medical cause for some non-specific and typically geriatric presentations (i.e., delirium, falls, hypokynesis). However, the majority of responders (68.1\%) declared that in their EDs objectives tools (i.e., scores) are not used in functional, cognitive and social evaluation of elderly patients, and only $26 \%$ of the responders considered that it may be useful to use these tools in daily routine.

Only $19.6 \%$ of the responders, with no differences between EPs and ENs, considered the health and social recordings accompanying the elderly patients referred to the ED by their general practitioner (GP) of good quality, and only $28.2 \%$ considered the health and social recordings accompanying the elderly patients referred to the ED by nursing homes of good quality. These last perceptions are of utmost importance for the future compelling development of simple but effective clinical recording and communication tools between GPs and

\section{Academy of Emergency Medicine and Care}

\section{Questionnaire on quality of ED elderly patients care.}

For the purposes of this questionnaire, "elderly" is defined as a patient of $>80 \mathrm{yrs}$, or, if aged $65-80 \mathrm{yrs}$, with defined frailty.

\section{Answer $\mathbf{1}=$ totally disagree; answer $\mathbf{5}=$ totally agree}

\begin{tabular}{|c|c|c|c|c|c|c|}
\hline & & 1 & 2 & 3 & 4 & 5 \\
\hline 1 & In your ED the elderly care has the same quality of adult care. & & & & & \\
\hline 2 & $\begin{array}{l}\text { Competence and communication attitudes of emergency health care } \\
\text { professionals are the same with elderly and adult patients. }\end{array}$ & & & & & \\
\hline 3 & $\begin{array}{l}\text { Health and social recordings of elderly patients referred to the ED by their } \\
\text { General Practitioners are generally of good quality. }\end{array}$ & & & & & \\
\hline 4 & $\begin{array}{l}\text { Health and social recordings of elderly patients referred to the ED by nursing } \\
\text { homes are generally of good quality. }\end{array}$ & & & & & \\
\hline 5 & $\begin{array}{l}\text { Always medical causes for non specific, typically geriatric, presentations (i.e., } \\
\text { delirium, falls, hypokynesis) are systematically investigated. }\end{array}$ & & & & & \\
\hline 6 & $\begin{array}{l}\text { The willingness to admit elderly patients in medical wards is the same as for } \\
\text { adult patients. }\end{array}$ & & & & & \\
\hline 7 & $\begin{array}{l}\text { During ED process basic needs of elderly patients (i.e., meals, hygiene, } \\
\text { micturition, evacuation) are always met. }\end{array}$ & & & & & \\
\hline 8 & $\begin{array}{l}\text { In your ED objectives tools (i.e., scores) for functional, cognitive and social } \\
\text { evaluation of elderly patients are generally used. }\end{array}$ & & & & & \\
\hline 9 & $\begin{array}{l}\text { In your opinion in daily routine it would be possible to use objectives tools (i.e., } \\
\text { scores) in functional, cognitive and social evaluation of elderly patients. }\end{array}$ & & & & & \\
\hline 10 & $\begin{array}{l}\text { It would be useful to use objectives tools (i.e., scores) for functional, cognitive } \\
\text { and social evaluation of elderly patients in daily routine? }\end{array}$ & & & & & \\
\hline 11 & $\begin{array}{l}\text { Before discharging an elderly patient, in your ED a quick functional, cognitive } \\
\text { and social evaluation is generally performed. }\end{array}$ & & & & & \\
\hline 12 & $\begin{array}{l}\text { The patient age influences by itself the triage priority code, or some different } \\
\text { priorities are given to the elderly if the triage code is the same. }\end{array}$ & & & & & \\
\hline 13 & $\begin{array}{l}\text { In your Hospital elderly-specific diagnostic and therapeutic post-discharge } \\
\text { pathways are generally provided. }\end{array}$ & & & & & \\
\hline 14 & In your Hospital the concept of "futility" is explicitly included in protocols & & & & & \\
\hline
\end{tabular}

(") with the term "futility" is generally expressed the uselessness of a diagnostic or therapeutic procedure, in terms of improvement of the health status of the patient

\section{Age I I I Gender $\mathrm{M} /[$ I $\mathrm{F} /]$ Role: Physician I_I Nurse I_I Auxiliary I_I}

Figure 1. The Academy of Emergency Medicine and Care questionnaire. 
EPs. Not surprisingly, $64.1 \%$ of the responders declared that elderly-specific diagnostic and therapeutic post-discharge pathways are not provided in their hospitals.

The last question, rather understandably, has been the most neglected (i.e., only 451 answers, representing $86 \%$ of the whole sample). The concept of medical futility has been a matter of debate for decades, and a univocal
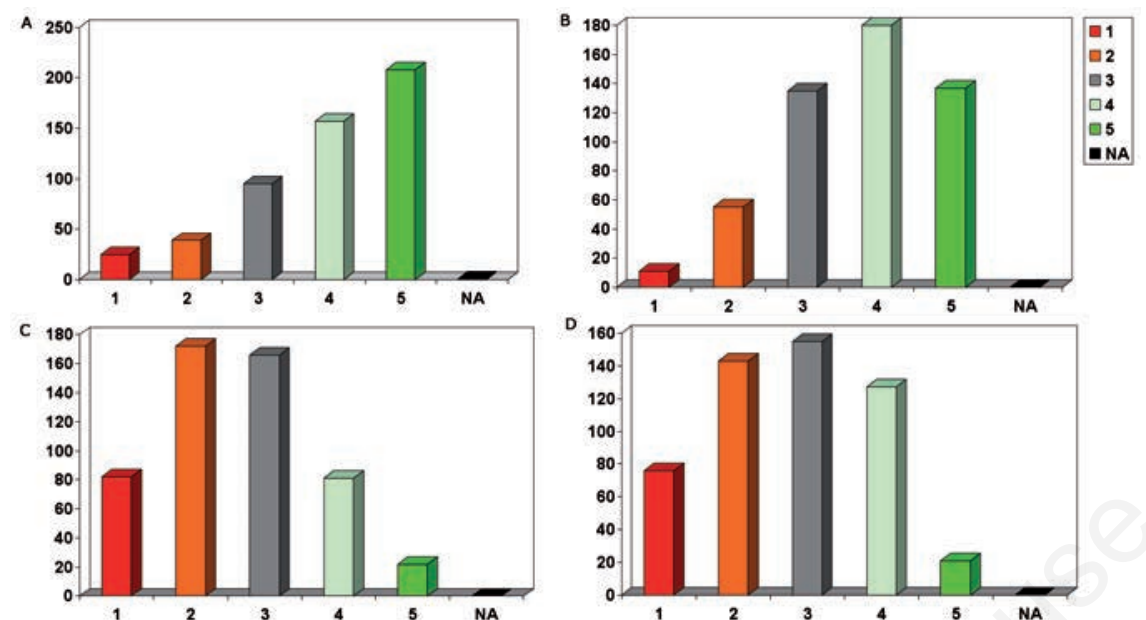

Figure 2. Answers to questions 1 (A; total $=523 ; 4+5$ scores=71.8\%); 2 (B; total=524; 4+5 scores $=61.7 \%) ; 3(C$; total $=524 ; 1+2$ scores $=49.1 \%) ; 4(D$; total $=524 ; 4+5$ scores $=28.2 \%)$. NA=not available.
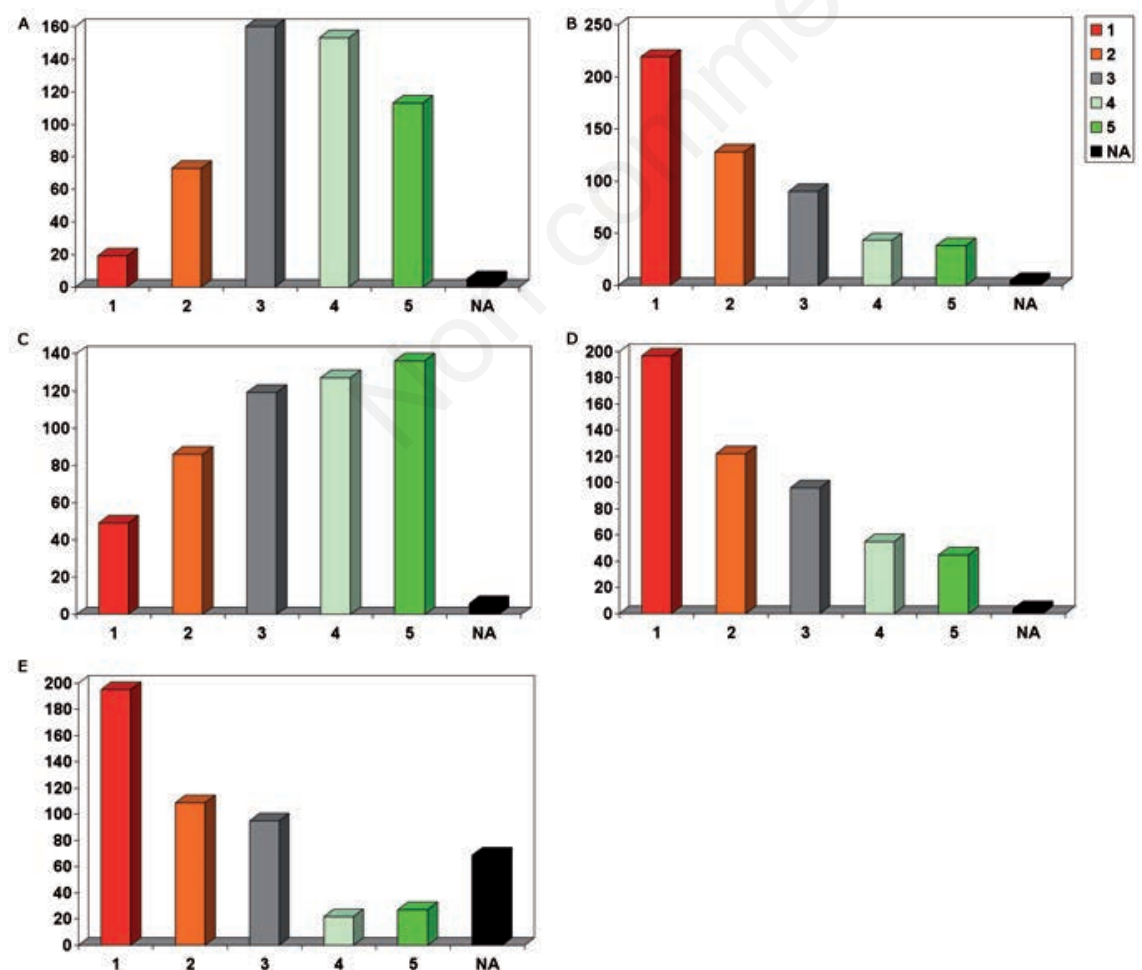

Figure 3. Answers to question 5 (A; total $=524 ; 4+5$ scores $=52.1 \%) ; 8(B$; total $=519 ; 1+2$ scores $=68.1 \%) ; 10(C ;$ total $=519 ; 1+2$ scores $=26.0 \%) ; 13(D ;$ total $=519 ; 1+2$ scores $=64.1 \%) ; 14($ total $=451 ; 1+2$ scores $=70.1 \%)$. NA=not available.

\section{Conclusions}

In conclusion, this is the first study focusing on the EPs' and ENs' perception of a broad spectrum of issues related to elderly emergency care, and the results describe an Italian community of EPs and ENs rather comfortable with geriatric emergency medicine, but largely unsatisfied with the healthcare out-of-hospital services. The lack of elderly-specific diagnostic and therapeutic post-discharge pathways is broadly appreciated, and the concept of medical futility is not explicitly included in the vast majority of Italian hospital protocols.

\section{References}

1. Palangkaraya A, Yong J. Population ageing and its implications on aggregate health care demand: empirical evidence from 22 OECD countries. Int $\mathrm{J}$ Health Care Fi 2009;9:391-402.

2. Mion LC, Palmer RM, Anetzberger GJ, et al. Establishing a casefinding and referral system for at-risk older individuals in the emergency department setting: the SIGNET model. J Am Geriatr Soc 2001;49:1379-86.

3. Samaras N, Chevalley T, Samaras D, Gold G. Older patients in the emergency department: a review. Ann Emerg Med 2010;56:261-9.

4. Salvi F, Morichi V, Grilli A, et al. The elderly in the emergency department: a critical review of problems and solutions. Intern Emerg Med 2007;2:292-301.

5. Lynne Grief C. Patterns of ED use and perceptions of the elderly regarding their emergency care: a synthesis of recent research. J Emerg Nurs 2003;29:122-6.

6. Shankar KN, Bhatia BK, Schuur JD. Toward patient-centered care: a systematic review of older adults' views of quality emergency care. Ann Emerg Med 2014; 63:529-50.

7. McNamara R, Rousseau E, Sanders AB. Geriatric emergency medicine: a survey of practicing emergency physicians. Ann Emerg Med 1992;21:796-800.

8. Sanders A. Emergency care of the elder person: instructors' manual. Introduction: principles of geriatric emergency medicine. Lansing (MI): Society for Academic Emergency Medicine; 1996.

9. Schneiderman LJ. Defining medical futility and improving medical care. J Bioeth Inq 2011;8:123-31. 